\title{
SPECIALIZATION OF MONODROMY GROUP AND $\ell$-INDEPENDENCE
}

\author{
CHUN YIN HUI
}

\begin{abstract}
Let $E$ be an abelian scheme over a geometrically connected variety $X$ defined over $k$, a finitely generated field over $\mathbb{Q}$. Let $\eta$ be the generic point of $X$ and $x \in X$ a closed point. If $\mathfrak{g}_{l}$ and $\left(\mathfrak{g}_{l}\right)_{x}$ are the Lie algebras of the $l$-adic Galois representations for abelian varieties $E_{\eta}$ and $E_{x}$, then $\left(\mathfrak{g}_{l}\right)_{x}$ is embedded in $\mathfrak{g}_{l}$ by specialization. We prove that the set $\left\{x \in X\right.$ closed point $\left.\mid\left(\mathfrak{g}_{l}\right)_{x} \subsetneq \mathfrak{g}_{l}\right\}$ is independent of $l$ and confirm Conjecture 5.5 in [2].
\end{abstract}

\section{$\S 0$. Introduction}

Let $E$ be an abelian scheme of relative dimension $n$ over a geometrically connected variety $X$ defined over $k$, a finitely generated field over $\mathbb{Q}$. If $K$ is the function field of $X$ and $\eta$ is the generic point of $X$, then $A:=E_{\eta}$ is an abelian variety of dimension $n$ defined over $K$. The structure morphism $X \rightarrow \operatorname{Spec}(k)$ induces at the level of étale fundamental groups a short exact sequence of profinite groups:

$$
1 \rightarrow \pi_{1}\left(X_{\bar{k}}\right) \rightarrow \pi_{1}(X) \rightarrow \Gamma_{k}:=\operatorname{Gal}(\bar{k} / k) \rightarrow 1
$$

Any closed point $x: \operatorname{Spec}(\mathbf{k}(x)) \rightarrow X$ induces a splitting $x: \Gamma_{\mathbf{k}(x)} \rightarrow \pi_{1}\left(X_{\mathbf{k}(x)}\right)$ of equation (0.1) for $\pi_{1}\left(X_{\mathbf{k}(x)}\right)$.

Let $\Gamma_{K}=\operatorname{Gal}(\bar{K} / K)$ the absolute Galois group of $K$. For each prime number $l$, we have the Galois representation $\rho_{l}: \Gamma_{K} \rightarrow \operatorname{GL}\left(T_{l}(A)\right)$ where $T_{l}(A)$ is the $l$-adic Tate module of $A$. This representation is unramified over $X$ and factors through $\rho_{l}: \pi_{1}(X) \rightarrow \operatorname{GL}\left(T_{l}(A)\right)$ (still denote the map by $\rho_{l}$ for simplicity). The image of $\rho_{l}$ is a compact $l$-adic Lie subgroup of $\mathrm{GL}\left(T_{l}(A)\right) \cong \mathrm{GL}_{2 n}\left(\mathbb{Z}_{l}\right)$. Any closed point $x: \operatorname{Spec}(\mathbf{k}(x)) \rightarrow X$ induces an $l$-adic Galois representation by restricting $\rho_{l}$ to $x\left(\Gamma_{\mathbf{k}(x)}\right)$. This representation is isomorphic to the Galois representation of $\Gamma_{\mathbf{k}(x)}$ on the $l$-adic Tate module of $E_{x}$, the abelian variety specialized at $x$.

For simplicity, write $G_{l}:=\rho_{l}\left(\pi_{1}(X)\right), \mathfrak{g}_{l}:=\operatorname{Lie}\left(G_{l}\right),\left(G_{l}\right)_{x}:=\rho_{l}\left(x\left(\Gamma_{\mathbf{k}(x)}\right)\right)$ and $\left(\mathfrak{g}_{l}\right)_{x}:=$ $\operatorname{Lie}\left(\left(G_{l}\right)_{x}\right)$. We have $\left(\mathfrak{g}_{l}\right)_{x} \subset \mathfrak{g}_{l}$. We set $X^{c l}$ the set of closed points of $X$ and define the exceptional set

$$
X_{\rho_{E, l}}:=\left\{x \in X^{c l} \mid\left(\mathfrak{g}_{l}\right)_{x} \subsetneq \mathfrak{g}_{l}\right\} .
$$

The main result (Theorem 1.4) of this note is that the exceptional set $X_{\rho_{E, l}}$ is independent of $l$. Conjecture 5.5 in [Cadoret \& Tamagawa 2] is then a direct application of our theorem.

\section{$\S 1$. l-independence of $X_{\rho_{E, l}}$}


Theorem 1.1. (Serre $[5 \S 1]$ ) Let $A$ be an abelian variety defined over a field $K$ finitely generated over $\mathbb{Q}$ and let $\Gamma_{K}=\operatorname{Gal}(\bar{K} / K)$. If $\rho_{l}: \Gamma_{K} \rightarrow \operatorname{GL}\left(T_{l}(A)\right)$ is the $l$-adic representation of $\Gamma_{K}$, then the Lie algebra $\mathfrak{g}_{l}$ of $\rho_{l}\left(\Gamma_{K}\right)$ is algebraic and the rank of $\mathfrak{g}_{l}$ is independent of the prime $l$.

Since $V_{l}:=T_{l}(A) \otimes_{\mathbb{Z}_{l}} \mathbb{Q}_{l}$ is a semisimple $\Gamma_{K}$-module (Faltings and Wüstholz [3 Chap. 6]), the action of the Zariski closure of $\rho_{l}\left(\Gamma_{K}\right)$ in $\mathrm{GL}_{V_{l}}$ is also semisimple on $V_{l}$. Therefore it is a reductive algebraic group (Borel [1]). By Theorem 1.1, $\mathfrak{g}_{l}$ is algebraic. So the rank of $\mathfrak{g}_{l}$ is just the dimension of maximal tori. We state two more theorems:

Theorem 1.2. (Faltings and Wüstholz [3 Chap. 6]) Let $A$ be an abelian variety defined over a field $k$ finitely generated over $\mathbb{Q}$ and let $\Gamma_{k}=\operatorname{Gal}(\bar{k} / k)$. Then the map $\operatorname{End}_{k}(A) \otimes_{\mathbb{Z}} \mathbb{Q}_{l} \rightarrow \operatorname{End}_{G_{k}}\left(V_{l}(A)\right)$ is an isomorphism.

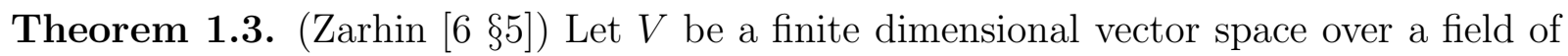
characteristic 0 . Let $\mathfrak{g}_{1} \subset \mathfrak{g}_{2} \subset \operatorname{End}(V)$ be Lie algebras of reductive subgroups of $\mathrm{GL}_{V}$. We assume that the centralizers of $\mathfrak{g}_{1}$ and $\mathfrak{g}_{2}$ in $\operatorname{End}(V)$ are equal and that the ranks of $\mathfrak{g}_{1}$ and $\mathfrak{g}_{2}$ are equal. Then $\mathfrak{g}_{1}=\mathfrak{g}_{2}$.

We are now able to prove our main theorem.

Theorem 1.4. The set $X_{\rho_{E, l}}$ is independent of $l$.

Proof. Suppose $x \in X^{c l} \backslash X_{\rho_{l}}$, then $\left(\mathfrak{g}_{l}\right)_{x}=\mathfrak{g}_{l}$. It suffices to show $\mathfrak{g}_{l^{\prime}}=\left(\mathfrak{g}_{l^{\prime}}\right)_{x}:=$ $\operatorname{Lie}\left(\rho_{l^{\prime}}\left(x\left(\Gamma_{\mathbf{k}(x)}\right)\right)\right)$ for any prime number $l^{\prime}$. Since base change with finite field extension of $\mathbf{k}(x)$ does not change the Lie algebras, $\operatorname{End}_{\bar{k}}\left(E_{x}\right)$ is finitely generated, and we have the exponential map from Lie algebras to Lie groups, we may assume that $\operatorname{End}_{\bar{k}}\left(E_{x}\right)=\operatorname{End}_{k}\left(E_{x}\right)$ and $\operatorname{End}_{\Gamma_{k}}\left(V_{l}\left(E_{x}\right)\right)=\operatorname{End}_{\left(\mathfrak{g}_{l}\right)_{x}}\left(V_{l}\left(E_{x}\right)\right)$. We do the same for the abelian variety $E_{\eta} / K$. We therefore have

$$
\begin{aligned}
& \operatorname{dim}_{\mathbb{Q}_{l^{\prime}}}\left(\operatorname{End}_{\mathfrak{g}_{l^{\prime}}}\left(V_{p}\left(E_{\eta}\right)\right)\right) \stackrel{1}{=} \operatorname{dim}_{\mathbb{Q}_{l^{\prime}}}\left(\operatorname{End}_{K}\left(E_{\eta}\right) \otimes_{\mathbb{Z}} \mathbb{Q}_{l^{\prime}}\right) \\
& \stackrel{2}{=} \operatorname{dim}_{\mathbb{Q}_{l}}\left(\operatorname{End}_{K}\left(E_{\eta}\right) \otimes_{\mathbb{Z}} \mathbb{Q}_{l}\right) \stackrel{3}{=} \operatorname{dim}_{\mathbb{Q}_{l}}\left(\operatorname{End}_{\mathfrak{g}_{l}}\left(V_{l}\left(E_{\eta}\right)\right)\right) \\
& \operatorname{dim}_{\mathbb{Q}_{l}}\left(\operatorname{End}_{\left(\mathfrak{g}_{l}\right)_{x}}\left(V_{l}\left(E_{x}\right)\right)\right) \stackrel{5}{=} \operatorname{dim}_{\mathbb{Q}_{l}}\left(\operatorname{End}_{k}\left(E_{x}\right) \otimes_{\mathbb{Z}} \mathbb{Q}_{l}\right) \\
& \operatorname{dim}_{\mathbb{Q}_{l^{\prime}}}\left(\operatorname{End}_{k}\left(E_{x}\right) \otimes_{\mathbb{Z}} \mathbb{Q}_{p}\right) \stackrel{7}{=} \operatorname{dim}_{\mathbb{Q}_{l^{\prime}}}\left(\operatorname{End}_{\left(\mathfrak{g}_{l^{\prime}}\right)_{x}}\left(V_{l^{\prime}}\left(E_{x}\right)\right)\right) .
\end{aligned}
$$

Theorem 1.2 implies the first, third, fifth and seventh equality. The dimensions of $\operatorname{End}_{K}\left(E_{\eta}\right) \otimes_{\mathbb{Z}}$ $\mathbb{Q}_{l}$ and $\operatorname{End}_{k}\left(E_{x}\right) \otimes_{\mathbb{Z}} \mathbb{Q}_{l}$ as vector spaces are independent of $l$ imply the second and the sixth equality. $\mathfrak{g}_{l}=\left(\mathfrak{g}_{l}\right)_{x}$ implies the fourth equality.

We have $\operatorname{End}_{\mathfrak{g}_{l^{\prime}}}\left(V_{l^{\prime}}\left(E_{\eta}\right)\right)=\operatorname{End}_{\left(\mathfrak{g}_{l^{\prime}}\right)_{x}}\left(V_{l^{\prime}}\left(E_{x}\right)\right)$ because the left one is contained in the right one. In other words, the centralizer of $\left(\mathfrak{g}_{l^{\prime}}\right)_{x}$ is equal to the centralizer of $\mathfrak{g}_{l^{\prime}}$. We know that $\left(\mathfrak{g}_{l^{\prime}}\right)_{x} \subset \mathfrak{g}_{l^{\prime}}$ are both reductive from the semisimplicity of Galois representaion (Faltings and 
Wüstholz [3 Chap. 6]). By Theorem 1.1 on $l$-independence of reductive ranks and $\mathfrak{g}_{l}=\left(\mathfrak{g}_{l}\right)_{x}$, we have:

$$
\operatorname{rank}\left(\mathfrak{g}_{l^{\prime}}\right)=\operatorname{rank}\left(\mathfrak{g}_{l}\right)=\operatorname{rank}\left(\mathfrak{g}_{l}\right)_{x}=\operatorname{rank}\left(\mathfrak{g}_{l^{\prime}}\right)_{x} .
$$

Therefore, by Theorem 1.3 we conclude that $\left(\mathfrak{g}_{l^{\prime}}\right)_{x}=\mathfrak{g}_{l^{\prime}}$ and thus prove the theorem.

Corollary 1.5 (Conjecture $5.5[2]$ ). Let $k$ be a field finitely generated over $\mathbb{Q}, X$ a smooth, separated, geometrically connected curve over $k$ with quotient field $K$. Let $\eta$ be the generic point of $X$ and $E$ an abelian scheme over $X$. Let $\rho_{l}: \pi_{1}(X) \rightarrow \operatorname{GL}\left(T_{l}\left(E_{\eta}\right)\right)$ be the $l$-adic representation. Then there exists a finite subset $X_{E} \subset X(k)$ such that for any prime $l, X_{\rho_{E, l}}=X_{E}$, where $X_{\rho_{E, l}}$ is the set of all $x \in X(k)$ such that $\left(G_{l}\right)_{x}$ is not open in $G_{l}:=\rho_{l}\left(\pi_{1}(X)\right)$.

Proof. The uniform open image theorem for GSRP representations [2 Thm. 1.1] implies the finiteness of $X_{\rho_{E, l}}$. Theorem 1.4 implies $l$-independence.

Corollary 1.6. Let $A$ be an abelian variety of dimension $n \geq 1$ defined over a field $K$ finitely generated over $\mathbb{Q}$. Let $\Gamma_{K}=\operatorname{Gal}(\bar{K} / K)$ denote the absolute Galois group of $K$. For each prime number $l$, we have the Galois representation $\rho_{l}: \Gamma_{K} \rightarrow \operatorname{GL}\left(T_{l}(A)\right)$ where $T_{l}(A)$ is the $l$-adic Tate module of $A$. If the Mumford-Tate conjecture for abelian varieties over number fields is true, then there is an algebraic subgroup $H$ of $\mathbf{G L}_{2 n}$ defined over $\mathbb{Q}$ such that $\rho_{l}\left(\Gamma_{K}\right)^{\circ}$ is open in $H\left(\mathbb{Q}_{l}\right)$ for all $l$.

Proof. There exists an abelian scheme $E$ over a variety $X$ defned over a number field $k$ such that the function field of $X$ is $K$ and $E_{\eta}=A$ where $\eta$ is the generic point of $X$ (see, e.g., Milne $[4 \S 20])$. By $[5 \S 1]$, there exists a closed point $x \in X$ such that $\left(\mathfrak{g}_{l}\right)_{x}=\mathfrak{g}_{l}$. Therefore, we have $\left(\mathfrak{g}_{l}\right)_{x}=\mathfrak{g}_{l}$ for any prime $l$ by Theorem 1.4. Since all Lie algebras are algebraic (Theorem 1.1), if we take $H$ as the Mumford-Tate group of $E_{x}, \rho_{l}\left(\Gamma_{K}\right)^{\circ}$ is then open in $H\left(\mathbb{Q}_{l}\right)$ for all $l$.

Question. Is the algebraic group $H$ in Corollary 1.6 isomorphic to the Mumford-Tate group of the abelian variety $A$ ?

Acknowledgement. This work grew out of an attempt to prove Conjecture 5.5 in [2] suggested by my advisor, Professor Michael Larsen. I am grateful to him for the suggestion, guidance and encouragement. I would also like to thank Anna Cadoret for her useful comments on an earlier version of this note.

\section{REFERENCES}

1. A. Borel, Linear Algebraic Groups, Graduate Texts in Mathematics, 126 (2nd ed.), Springer-Verlag 1991.

2. A. Cadoret and A. Tamagawa, A uniform open image theorem for l-adic representations I, preprint.

3. G. Faltings and G. Wüstholz, Rational Points, Seminar Bonn/Wuppertal 1983-1984, Vieweg 1984.

4. J. S. Milne, "Jacobian Varieties". Arithmetic Geometry. New York: Springer-Verlag 1986.

5. J-P. Serre, Letter to K. A. Ribet, Jan. 1, 1981.

6. Y. G. Zarhin, Abelian varieties having a reduction of K3 type, Duke Math. J. 65 (1992), 511-527. 
Department of Mathematics, Indiana University, Bloomington, IN 47405, USA E-mail address: chhui@umail.iu.edu 